\title{
Evaluation of Aggressive Behavior and Invasive Features of Pituitary Adenomas Using Radiological, Surgical, Clinical and Histopathological Markers
}

\author{
Ahmet KUCUK ${ }^{1}$, Fahri BAYRAM ${ }^{2}$, Figen OZTURK ${ }^{3}$, Abdulfettah TUMTURK ${ }^{1}$, Halit DIRI ${ }^{2}$, Sukru ORAL ${ }^{1}$, \\ Bulent TUCER ${ }^{1}$, Ahmet Candan DURAK ${ }^{4}$, Ali KURTSOY ${ }^{1}$ \\ ${ }^{1}$ Erciyes University, School of Medicine, Department of Neurosurgery, Kayseri, Turkey \\ ${ }^{2}$ Erciyes University, School of Medicine, Department of Endocrinology, Kayseri, Turkey \\ ${ }^{3}$ Erciyes University, School of Medicine, Department of Pathology, Kayseri, Turkey \\ ${ }^{4}$ Erciyes University, School of Medicine, Department of Radiology, Kayseri, Turkey
}

\section{ABSTRACT}

AIM: Total surgical resection of pituitary macroadenomas is difficult due to the location of the adenoma and the propensity to invade surrounding tissues. The purpose of this study was to evaluate the risk factors for invasive and aggressive pituitary macroadenomas using radiological, hormonal, clinical, and immunohistochemical markers.

MATERIAL and METHODS: Seventy cases of pituitary macroadenoma were examined. Age, gender, symptoms, the presence of fibrosis within the adenoma, hormonal levels, radiological findings, pathological results and immunohistochemical staining of the patients were evaluated using statistical methods.

RESULTS: We observed that the patients with macroadenomas in our study most frequently presented during their $5^{\text {th }}$ decade. The most frequent pituitary adenomas were non-functional, GH-secreting and PRL-secreting macroadenomas. The most frequent complaint was vision loss, headache and acral growth. Based on Magnetic Resonance Imaging (MRI) results, it was observed that the degree of invasion into surrounding tissues increased as the size of the macroadenoma increased. Macroadenomas that had invaded into the cavernous sinus invasion or that had a fibrotic tumor structure had a low probability of being resectable. There were no significant relationships between invasive behavior and p53, telomerase, ghrelin and CD46.

CONCLUSION: It is not possible to identify only one factor that affects the prognosis of patients with pituitary macroadenomas. The contribution of the experience of surgeon to the treatment is surely beyond dispute. Fibrotic tumor structure, the surgical technique, the type of hormone, and cavernous sinus invasion affect the ability to perform a total resection and the overall prognosis.

KEYWORDS: Pituitary adenoma, Aggressive behavior, Invasion, Immunohistochemical marker

\section{INTRODUCTION}

$\mathrm{P}$ ituitary adenomas are located in the bone complex called the 'Sella Turcica', which means 'Turkish Saddle', and are the most frequent brain tumors after gliomas and meningiomas. Pituitary adenomas account for 10$15 \%$ of all primary brain tumors (16). The prevalence in the developed countries is around 1:1000 (2). Pituitary adenomas are clinically, pathologically and endocrinologically different from other intracranial tumors. Pituitary adenomas are slowgrowing, benign tumors and some can expand beyond the sella and invade the optic chiasm, cavernous sinus, sphenoid sinus and surrounding areas (1). Infiltration of the adenoma into 
the cavernous sinus can be followed by invasion through bone into other regions of the brain in some cases (13). There are no known predictors of the likelihood of aggressive behavior or relapse for this type of tumor. There are ongoing discussions about the relationship between cavernous sinus expansion and cavernous sinus medial wall defects and tumor biology (5). Recent genetic studies have not been able to identify a factor predicting the degree of invasion and aggressive behavior of these tumors $(4,6,12,20,27)$. The current idea is that the biological behavior of tumor is a significant factor. The presence of fibrosis within the adenoma negatively affects the likelihood of total resection (27).

In this study, age, gender, symptoms, shape of the adenoma, size of the adenoma, pathological results, cavernous sinus invasion, whether the structure of the adenoma was fibrotic, resection rates, hormonal values, and biomarkers not routinely measured for pituitary adenomas such as p53, ghrelin, telomerase and CD46 with routine immunohistochemical staining features of the cases with pituitary macroadenoma were examined. The relationship of these factors with each other and with the prognosis, aggressive behavior and degree of invasion of pituitary macroadenomas was determined.

\section{- MATERIAL and METHODS}

In this study, 100 surgeries performed in 70 patients with pituitary macroadenomas at the Erciyes University, Medical Faculty, Department of Neurosurgery Surgery between 2004 and 2011 were examined. Our study was supported by the Erciyes University, Unit of Scientific Research Projects. This study was approved by the Erciyes University, Medical Faculty, Clinical Studies Ethical Evaluation Commission.

For each case, we filled out "The Monitor Form of Pituitary Adenoma with Invasion and Aggressive Behavior". The cases were evaluated by the Neurosurgery--EndocrinologyRadiology Council and treated using proper surgical techniques after pre-surgical evaluation was completed. Age, gender, hormone levels, and symptoms of patients, type of tumors, pre-surgery and post-surgery imaging results, postoperative complications, and immunohistochemical staining features were examined.

All patients received routine $T 1$, pre-contrast sagittal and coronal pituitary magnetic resonance imaging (MRI) and postcontrast sagittal and coronal MRI before the surgery. In some cases, two or more surgeries were performed. In total, 100 surgeries were performed, 76 of which were transsphenoidal and 24 of which were transcranial.

\section{Evaluation of Cavernous Sinus Invasion Using MRI}

In this study, the pituitary adenoma invasion rates were calculated using the method by Knosp, which looks at the relationship between the adenoma and the carotid artery supra- and intra-cavernous segments (15).

\section{Immunohistochemical Methods}

Pituitary adenoma specimens were stained with LH, FSH, prolactin, $\mathrm{ACTH}, \mathrm{GH}$, and TSH antibodies in addition to routine immunohistochemical stains. Additionally, the specimens were stained for telomerase, ghrelin, p53, and CD46 in order to evaluate the aggressiveness of the tumor. Resected tissues were first kept at $60^{\circ} \mathrm{C}$ for 1 hour. The tissues were incubated in xylol for 15 minutes and then in 100\%, 95\%, and $70 \%$ alcohols for 5 minutes each. Then, the tissue was incubated in distilled water for 10 minutes. The tissues were placed into citrate buffer and were heated in a microwave oven for 20 minutes and left to cool in distilled water for 5 minutes. The tissues were then soaked in $3 \% \mathrm{H}_{2} \mathrm{O}_{2}$ for 10 minutes. Then the tissues were rinsed in distilled water for 5 minutes and then in Phosphate Buffer Solution (PBS) for 5 minutes. After these steps the tissues were incubated in the primary antibody for 1-24 hours, followed by rinsing with PBS for 10 minutes. The tissues were incubated in biotin for 10 minutes and then rinsed again in PBS for 10 minutes. Thereafter, they were incubated in streptavidin for 10 minutes and then rinsed in PBS for 10 minutes. The tissues were incubated with chromogen and then rinsed in distilled water for 10 minutes. Then, they were incubated in hematoxylin of Meyer for 2 minutes and then rinsed in distilled water, $70 \%, 96 \%$ alcohol, $100 \%$ alcohol and xylol for 5 minutes each. The specimen was secured to the glass with balsam before the tissues were dried. The degree of staining with telomerase and ghrelin was classified as follows: $<10 \%=$ negative staining, $10-50 \%=$ light staining, $50-90 \%=$ medium staining, $>90 \%=$ heavy staining. For $p 53$, the staining classification was as follows: very few cells staining with $\mathrm{p} 53=$ rare staining, $<50 \%$ cells positive for $p 53$ staining $=$ isolated staining, $>50 \%$ cells positive for p53 staining= widespread staining. For CD46, specimens that were $0-50 \%$ positive for CD46 staining was classified as weak staining and specimens with greater than $50 \%$ CD46 staining were classified as strong staining.

\section{Statistical Methods}

The data were expressed as average, standard deviation, frequency and rate. The Kolmogrov-Smirnov test was used to determine the distribution of the variables. The homogeneity of the variances of the variables was tested. Student's t-test and ANOVA (Tukey test in the sub-analysis) were used in to analyze parametric variables. The Mann-Whitney $U$ test and Kruskal-Wallis test (Mann-Whitney $U$ test in the sub-analysis) were used to analyze non-parametric variables. A paired sample test was used in the repeating measurement analysis of the parametric data, and the Wilcoxon test was used in the repeating measurement analysis of the non-parametric data. The Chi-squared test was used to analyze categorical data; if the Chi-squared test conditions were not met, the Fisher test was used. SPSS 19.0 was used for statistical analysis. All analyses were done using a confidence interval of $95 \%$.

\section{RESULTS}

Twenty-five of the 70 patients were female (35.7\%) and 45 were male $(64.3 \%)$, and the average age was 46.1 (range=19-77) years. The average period that the patients were followed was 24.5 (range $=6-67$ ) months. It was observed that pituitary macroadenomas were most common in women aged 50-59 years and men aged $40-49$ years. Thirty-eight cases (54.2\%) 
had non-functional adenomas, 15 cases (21.4\%) had GHsecreting adenomas, 14 cases $(20 \%)$ had prolactin-secreting adenomas, 2 cases $(2.9 \%)$ had ACTH-secreting adenomas and 1 case (1.5\%) had a TSH-secreting adenoma (Table I).

The most frequent symptoms were loss or decrease in vision in 27 cases (38\%), headache in 18 cases $(25.3 \%)$, acral growth in 15 cases $(21.1 \%)$, amenorrhea in 4 cases (5.6\%), dizziness in 3 cases $(4.2 \%)$, galactorrhea in 1 case $(1.5 \%)$, erectile dysfunction in 2 cases $(2.8 \%)$ and gaining weight in 1 case $(1.5 \%)$.

Unilateral cavernous sinus invasion was observed in $34 \mathrm{MRIs}$ $(48.5 \%)$ and bilateral cavernous sinus invasion was seen in 17 MRIs (24.2\%).

Fifty of the patients (71.4\%) required only one operation, 14 $(20 \%)$ required two operations, two $(2.9 \%)$ required three operations and four (5.7\%) required four operations. Therefore, 100 surgeries were performed in 70 patients. When the cases with one surgery and the cases with 2 or more surgeries were compared, there was no significant difference in terms of total resection $(p=0.179)$. Seventy-six of the 100 surgeries $(76 \%)$ were transsphenoidal and 24 (24\%) were transcranial. There was no significant difference between the surgical approach and total resection $(p>0.05)$. Out of the 51 cases with internal carotid artery invasion seen on MRI, 10 (19.6\%) had successful removal of the invading tumor and residual tumor was seen in the remaining 41 cases $(80.4 \%)$.

When the immunohistochemical data was evaluated, 69 cases $(98.6 \%)$ had rare staining for p53, and only one case $(1.4 \%)$ had isolated staining $(<50 \%)$ for p53 and no cases had widespread ( $>50 \%$ ) p53 staining. Up to $10 \%$ staining for telomerase was detected in 29 cases (41.4), 10-50\% staining for telomerase was seen in 9 cases (12.9\%), 50-90\% staining was seen in 10 cases (14.3\%) and $>90 \%$ staining was seen in 22 cases $(31.4 \%)$. Up to $10 \%$ staining for ghrelin was seen in 32 cases $(45.7 \%), 10-50 \%$ staining was seen in 14 cases (20\%), $50-90 \%$ staining was seen in 19 cases $(27.1 \%)$ and $>90 \%$ staining was seen in 5 cases $(7.1 \%)$. Weak staining for CD46 was seen in 24 cases (34.2\%), medium staining was detected in 25 cases (35.7\%) and strong staining was detected in 21 cases (30\%) (Table II).

There was a significant difference in the telomerase staining between cases with and without cavernous sinus invasion $(p=0.015$, Table III). In the cases that had $>50 \%$ staining for telomerase, 15 (\%48.3) were non-functional adenomas, $10(32.2 \%)$ were $\mathrm{GH}$-secreting adenomas, 6 (19.3\%) were prolactinomas.

There was no statistically significant difference in ghrelin staining between cases with and without cavernous sinus invasion ( $p=0.271$, Table IV). Nine of the cases with $>50 \%$ ghrelin antibody staining (41\%) were $\mathrm{GH}$-secreting adenomas, $9(41 \%)$ were non-functional adenomas, 3 (13.5\%) were prolactinomas, and 1 was a ACTH-secreting adenoma (4.1\%).

There was no statistically significant difference in CD46 staining between cases with and without cavernous sinus invasion ( $p=0.482$, Table V). Eleven of the cases (\%52.3) with $>50 \%$ CD46 staining were non-functional adenomas, five (23.8\%) were $\mathrm{GH}$-secreting adenomas and five $(23.8 \%)$ were prolactinomas.

Table I: Type of Adenoma in Male and Female Cases

\begin{tabular}{lcccc}
\hline Adenoma type & Female & Male & Total & Rate \\
\hline Non-functional adenoma & 12 & 26 & 38 & $54.2 \%$ \\
\hline GH-secreting adenoma & 7 & 8 & 15 & $21.4 \%$ \\
\hline PRL-secreting adenoma & 4 & 10 & 14 & $20 \%$ \\
\hline ACTH-secreting adenoma & - & 2 & 2 & $2.9 \%$ \\
\hline TSH-secreting adenoma & 1 & - & 1 & $1.5 \%$ \\
\hline
\end{tabular}

Table II: Pituitary Adenoma Staining Features according to Tumor Type

\begin{tabular}{|c|c|c|c|c|c|c|c|c|c|c|c|c|c|c|}
\hline \multirow[b]{2}{*}{ Staining Rate } & \multicolumn{3}{|c|}{ p53 } & \multicolumn{4}{|c|}{ Telomerase } & \multicolumn{4}{|c|}{ Ghrelin } & \multicolumn{3}{|c|}{ CD46 } \\
\hline & 0 & 1 & 2 & 0 & 1 & 2 & 3 & 0 & 1 & 2 & 3 & 0 & 1 & 2 \\
\hline Non-functional & 38 & 1 & - & 18 & 4 & 6 & 10 & 21 & 5 & 7 & 3 & 17 & 11 & 10 \\
\hline GH-secreting & 15 & - & - & 4 & 1 & 1 & 9 & 2 & 3 & 8 & 2 & 2 & 8 & 5 \\
\hline PRL-secreting & 14 & - & - & 5 & 3 & 3 & 3 & 7 & 4 & 3 & - & 4 & 4 & 6 \\
\hline ACTH-secreting & 2 & - & - & 1 & 1 & - & - & - & 1 & 1 & - & 1 & 1 & - \\
\hline TSH-secreting & 1 & - & - & 1 & - & - & - & - & 1 & - & - & - & 1 & - \\
\hline Total & 69 & 1 & - & 29 & 9 & 10 & 22 & 32 & 14 & 19 & 5 & 24 & 25 & 21 \\
\hline
\end{tabular}




\section{DISCUSSION}

In this study, the most frequent adenoma based on histopathology was a non-functional adenoma that was present in 38 out of 70 cases (54.2\%). A GH-secreting adenoma was present in 15 of the 70 cases $(21.4 \%)$ and a PRL-secreting adenoma was present in 14 cases (20\%). In the study of 2091 pituitary adenomas by Horvarth et al., PRL-secreting adenomas were seen most frequently (27\%), followed by the oncocytomas (non-functional adenoma) (13.4\%), GH-secreting adenomas (13.3\%), and null-cellular adenomas (12.4\%) (11). The reason for the difference in the results between our study and the Horvarth et al. study may be that we only had cases with macroadenomas in our study. In the study by Osamura et al., which included 382 cases, non-functional adenomas were the most common (31.5\%), followed by $\mathrm{GH}$-secreting adenomas (26.8\%), PRL-secreting adenomas (17.8\%) and multi-hormonal secreting adenomas (10.6\%) (22).
In the literature, the rate of cavernous sinus invasion by pituitary adenomas is $15-20 \%(7,9)$. As the adenoma grows, it extends into surrounding tissues and frequently may invade the cavernous sinus. The invasion may be either unilateral or bilateral. Once cavernous sinus invasion is present, it is difficult to perform a complete resection of the adenoma and thus, only a partial remission may be achieved. Relatedly, in our study, unilateral cavernous sinus invasion was observed in 34 cases (48.5\%), and bilateral cavernous sinus invasion in 17 cases (24.2\%). In the study by Pan et al., in which cavernous sinus invasion was examined in patients with pituitary adenomas using MRI and biological markers, cavernous sinus invasion was found in 25 cases (55.5\%), and bilateral cavernous sinus invasion was present in 5 of those cases (11.1\%) (23). In our study, cavernous sinus invasion was relatively more common, probably because we were only studying macroadenomas. In the study conducted by Farooq et al., cavernous sinus invasion was detected in 57 of the 100 macroadenoma cases $(57 \%)$ with no mention of whether the invasion was unilateral

Table III: The Relationship Between Telomerase Staining and Cavernous Sinus Invasion

\begin{tabular}{|c|c|c|c|c|c|c|c|}
\hline & & \multirow{2}{*}{$\begin{array}{c}\text { Cavernous } \\
\text { Staining Rate }\end{array}$} & \multicolumn{2}{|c|}{ Invasion (+) } & \multicolumn{2}{|c|}{ Invasion(-) } & \multirow[b]{2}{*}{$p$-value } \\
\hline & & & $\mathbf{n}$ & $\%$ & $\mathrm{n}$ & $\%$ & \\
\hline \multirow{4}{*}{ Telomerase } & \multirow{2}{*}{$0-50 \%$} & $10 \%$ & 4 & 20 & 26 & 52 & \multirow{4}{*}{0.015} \\
\hline & & $10-50 \%$ & 2 & 10 & 7 & 14 & \\
\hline & \multirow{2}{*}{$>50 \%$} & $50-90 \%$ & 3 & 15 & 6 & 12 & \\
\hline & & $>90 \%$ & 11 & 55 & 11 & 22 & \\
\hline
\end{tabular}

Chi-Square Test 95\% confidence interval

Table IV: The Relationship Between Ghrelin Staining and Cavernous Sinus Invasion

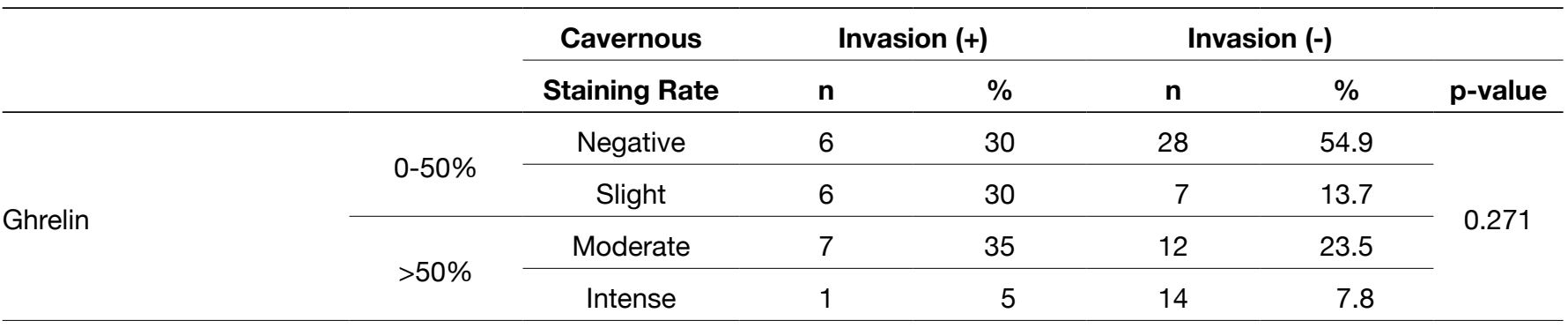

Chi-Square test $95 \%$ confidence interval

Table V: The Relationship Between CD46 Staining and Cavernous Sinus Invasion

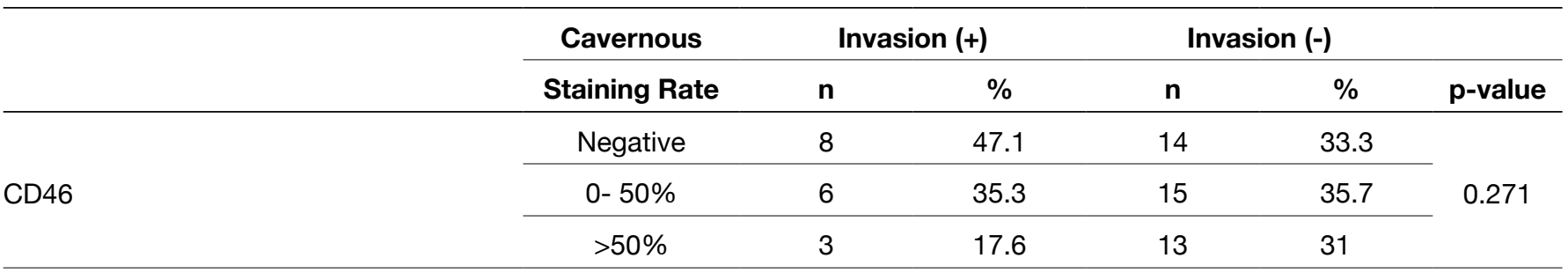

Chi-Square Test $95 \%$ confidence interval 
or bilateral (8). These findings support the idea that cavernous sinus invasion is seen more frequently in macroadenomas. When cavernous sinus invasion is present, there is a decrease in the rate of successful total resections. In our study, subtotal resection was performed in 37 of 51 macroadenoma cases with cavernous sinus invasion (72\%) and total resection could be performed in only 14 cases $(28 \%)$. Total resection could not be performed in 30 cases with cavernous sinus invasion in the study by Pan et al. (23). In cases without cavernous sinus invasion, total resection could be performed in 17 of the 20 cases (85\%) and in the remaining three cases $(15 \%)$, subtotal resection was performed. The total resection rate in the cases without cavernous sinus invasion is significantly higher than in cases with invasion $(p=0.016)$.

Another factor influencing the ability to perform total resection is the experience of the surgeon and surgical team. In order to minimize complications and the need for second operations, it is essential to choose experienced surgeons.

An experienced pituitary surgeon should meet the following criteria:

\section{a. He / She should perform at least 50 pituitary operations per year}

b. He/She should work with an endocrinologist, neuropathologist and radiation oncologist (21).

The surgeries in this study were performed by a team experienced in pituitary surgery. Our team has operated on 400 pituitary adenomas and performs $30-40$ pituitary surgeries per year.

In cases with cavernous sinus invasion, it is likely that some residual tumor will be present post-operatively. If the pituitary adenoma is hormonally active, the residual tumor tissues will continue to secrete hormone. It is likely that the residual tumor will continue to exhibit aggressive behavior, and may even enlarge to the point that a secondary operation is required. Additionally, invasive pituitary adenomas may require medical treatment after surgery. Therefore, it is essential to identify and know the extent of cavernous sinus invasion prior to surgery in order to determine the best surgical approach and postsurgical care.

A pituitary adenoma that has progressed to a fibrotic adenoma cannot be completely resected. It is difficult to aspirate a fibrotic adenoma and may be impossible to biopsy with cavitron ultrasonic aspirators (CUSA). In our study, three out of the 70 cases were fibrotic adenomas. While there is no clear data about this subject in the literature, the study by Snow et al. examined the efficacy of MRI for determining the surgical approach for large pituitary adenomas and found that macroadenomas with a T2A hyper-intense signal on MRI were more soft and thus, more easily aspirated during surgery (27). In contrast, fibrotic adenomas, which are hard and difficult to aspirate, had a hypo-intense T2A MRI signal. Two out of the three cases of fibrotic adenomas in our study had T2A hypointense images on MRI.

There are numerous studies on the relationship between the ability of pituitary adenomas to invade the cavernous sinus and biological markers. In these studies, there were some associations with certain biomarkers, but no significant results were obtained with other biomarkers. In fact, doctors are not able to counsel patients on how aggressive their pituitary tumor is likely to be based on biomarker data at this point. Ki-67 is one of the most important immunocytochemical markers of proliferation and it is used in the routine histological evaluation of different tumors (18). The correlation between Ki-67 expression and tumor recurrence, regrowth, and invasiveness has been demonstrated by several authors $(10,19,24,29)$. One of the most important results of our study may be that only one case stained $<50 \%$ with the p53 antibody and all other cases had even less staining. In the study by Blevins et al., they stated that p53 expression was related to invasion and aggressive behavior in pituitary adenomas and that this relationship was even stronger in pituitary macroadenomas (3). In the study by Thapar et al., the correlation between p53 expression and invasivity of pituitary adenomas and carcinomas was examined, and p53 was positive in only 5 of the 33 invasive cases (15\%) (28). In the study by Karabaglı, 9 of the 28 invasive pituitary adenomas (32.1\%) stained positive for p53. They concluded that p53 was more likely to be positive in aggressive pituitary adenomas, particularly in relapsing adenomas with a high degree of invasion (13). In our study, only one case stained mildly positive for p53 and this may indicate that p53 is not a good marker of invasive behavior in pituitary macroadenomas.

When we examined our cases with positive staining for telomerase, there was a significant difference in terms of staining with telomerase and cavernous sinus invasion $(p=0.015)$. Macroadenomas that did not invade the cavernous sinus showed more staining for telomerase. In the study in which Plata et al. examined the relationships between telomerase, PCNA and CD34 in pituitary adenomas, 14 out of 49 cases (28.5\%) stained positive for telomerase, additionally, hTERT (human telomerase reverse transcriptase), which is the catalytic portion of telomerase, may be a good marker for hormonally active pituitary adenomas that have increased cellular proliferation and tend to present at an early age (25). In our study, 31 out of 70 cases (43.6\%) had $>50 \%$ positive staining for telomerase. Due to the fact that staining with telomerase was more significant in the cases without cavernous sinus invasion compared to cases with cavernous sinus invasion, telomerase cannot be used as a marker for cavernous sinus invasion.

Ghrelin is a 28 amino acid hormone that affects the secretion of growth hormone and plays a role in energy balance and food intake. It is released into the blood stream by A-like cells in the oxyntic glands in the stomach; however, it is also released at lower levels in other parts of the body. Based on past studies, ghrelin has many functions (3). Ghrelin can also have an immunopositivity in neuroendocrine tumors including pituitary tumors (14). There are few past studies on ghrelin and pituitary adenomas. In a study of 107 cases by Rotondo et al., ghrelin levels in the normal pituitary and pituitary adenomas were examined and they found that ghrelin staining had differing prevalence and intensity in pituitary adenoma subtypes (26). However, these results were not 
significant when taking into account age, gender, tumor size, body weight and hormone profile and thus they concluded that ghrelin immunopositivity in pituitary adenomas cannot be used as a biomarker for cell proliferation, biological behavior, response to treatment and prognosis. We also examined the relationship between cavernous sinus invasion and ghrelin. There was no statistically significant difference in terms of staining with ghrelin, when separated into staining $<50 \%$ and $>50 \%$, and the presence of cavernous sinus invasion $(p=0.489)$. Therefore, we also conclude that ghrelin cannot be used as a biomarker for cavernous sinus invasion in pituitary adenomas.

CD46, also known as membrane co-factor protein, is a complement regulatory protein. CD46 is detected in all nuclear cells stimulated by the autologous complement system. There has been an increased interest in CD46 in recent years. The relationship between CD46 and tumors has been examined. While no significant results were obtained in the studies of mesothelioma and colon cancer, the study by Madjid et al. showed that 507 out of $510(99.4 \%)$ of patients with breast cancer had positive CD46 staining in the parenchyma of the tumor (17). Intense staining was observed in 136 of these cases $(27 \%)$, and moderate staining was observed in 222 of these cases (43\%). As a result of that study, a significant relationship between CD46 and histological classification of breast cancer $(p=0.003)$, tumor size $(p=0.034)$ and recurrence $(p=0.007)$ has been identified. Our study is the first in literature to examine CD46 and pituitary adenomas. However, there was no statistically significant relationship between the CD46 and cavernous sinus invasion $(p=0.496)$. Thus, CD46 cannot be used as a marker for cavernous sinus invasion in pituitary adenomas.

\section{CONCLUSION}

In this study, we examined the aggressive and invasive behavior of pituitary macroadenomas. This study only looked at macroadenomas and all surgery was performed by experienced surgeons. We examined the relationship between CD46, p53, telomerase and ghrelin and cavernous sinus invasion by the pituitary macroadenoma. There are very few studies in the literature on this subject. We found that none of these biomarkers were good predictors of pituitary macroadenoma aggressive and invasive behavior. Further studies with more cases are needed to identify potential biomarkers for aggressive and invasive behavior in pituitary adenomas, particularly in macroadenomas.

\section{- REFERENCES}

1. Asa SL: Practical pituitary pathology: What does the pathologist need to know? Arch Pathol Lab Med 132:12311240, 2008

2. Beckers A, Aaltonen LA, Daly AF, Karhu A: Familial isolated pituitary adenomas (FIPA) and the pituitary adenoma predisposition due to mutations in the aryl hydrocarbon receptor interacting protein (AIP) gene. Endocr Rev 34:239277,2013
3. Bilgin HM: Ghrelin; The renown hormone. Dicle Med J 33:268272, 2006

4. Blevins LS Jr, Verity DK, Allen G: Aggressive pituitary tumors. Oncology-NY 12:1307-1312, 1998

5. Ceylan S, Anık I, Koc K: A new endoscopic surgical classification and invasion criteria for pituitary adenomas involving the cavernous sinus. Turk Neurosurg 21:330-339, 2011

6. Cuny $T$, Pertuit $M$, Sahnoun-Fathallah $M$, Daly $A F$, Occhi G, Odou MF, Tabarin A, Nunes ML, Delemer B, Rohmer V, Desailloud R, Kerlan V, Chabre O, Sadoul JL, Cogne M, Caron P, Cortet C, Lienhardt-Roussie A, Raingeard I, Guedj AM, Brue T, Beckers A, Weryha G, Enjalbert A, Barlier A: Genetic analysis in young patients with sporadic pituitary macroadenomas: Beside AIP don't forget MEN1 genetic analysis. Eur J Endocrinol 168:533-541, 2013

7. Fahlbusch R, Buchfelder M, Nomikos P: Management options in the treatment of invasive pituitary tumors. In: Eisenberg MB, Al-Mefty O (eds). The Cavernous Sinus: A Comprehensive Text. Philadelphia: Lippincott Williams and Wilkins, 2000:291295

8. Farooq K, Rashid A, Malik TG: Pituitary macroadenomas: Demographic, visual and neuro-radiological patterns. Professional Med J 17: 623-627, 2010

9. Frank G, Pasquini E: Endoscopic endonasal cavernous sinus surgery, with special reference to pituitary adenomas. Front Horm Res 34:64-82,2006

10. Gejman R, Swearingen B, Hedly-Whyte T: Role of Ki67 proliferation index and p53 expression in predicting progression of pituitary adenomas. Hum Pathol 39:758-766, 2008

11. Horvath E, Scheithauer BW, Kovacs K, Lloyd RV: Hypothalamus and pituitary. In: Graham DI, Lantos PL (eds). Greeenfield's Neuropathology, 7th ed. London: Edward Arnold, 2002:983-1062

12. Jia W, Lu R, Jia G, Ni M, Xu Z: Expression of pituitary tumor transforming gene (PTTG) in human pituitary macroadenomas. Tumor Biol 34:1559-1567, 2013

13. Karabaglı $\mathrm{H}$, Karabaglı $\mathrm{P}$ : Expression of p53 protein in the clinical behavior of invasive pituitary adenomas. Türk Nöroşir Derg 18:42-47, 2008

14. Kim K, Arai K, Sanno K, Osamura Y, Teramoto A, Scibasaki $\mathrm{T}$ : Ghrelin and growth hormone $(\mathrm{GH})$ secretagogue receptor (GHSR) mRNA expression in human pituitary adenomas. Clin Endocrinol (Oxf) 54:759- 768, 2001

15. Knosp E, Steiner E, Kitz K: Pituitary adenomas with invasion of the cavernous sinus space: A magnetic resonance imaging classification compared with surgical findings. Neurosurgery 33:610-618, 1993

16. Kovacs $\mathrm{KH}$, Horvath $\mathrm{E}$ : Tumors of the pituitary gland. Washington DC: Armed Forces Institute of Pathology, 1986

17. Madjd Z, Durrant LG, Pinder SE, Ellis IO, Ronan J, Lewis S, Rushmere NK, Spendlov I: Do poor-prognosis breast tumours express membrane cofactor proteins (CD46)? Cancer Immunol Immunother 54:149-156, 2005 
18. Mastronardi L, Guiducci A, Puzzilli F: Lack of correlation between Ki-67 labelling index and tumor size of anterior pituitary adenomas. BMC Cancer 1:12, 2001

19. Mastronardi L, Guiducci A, Spera C, Puzzili F, Liberati F, Maira G: Ki-67 labelling index and invasiveness among anterior pituitary adenomas: Analysis of 103 cases using the MIB-1 monoclonal antibody. J Clin Pathol 52:107-111, 1999

20. McCabe CJ, Khaira JS, Boelaert K, Heaney AP, Tannahill LA, Hussain S, Mitchell R, Olliff J, Sheppard MC, Franklyn $\mathrm{JA}$, Gittoes NJ: Expression of pituitary tumour transforming gene (PTTG) and fibroblast growth factor-2 (FGF-2) in human pituitary adenomas: Relationships to clinical tumour behaviour. Clin Endocrinol (Oxf) 58:141-150, 2003

21. Melmed S, Colao A, Barkan A, Molitch M, Grossman AB, Kleinberg D, Clemmons D, Chanson P, Laws E, Schlechte J, Vance ML, Ho K, Giustina A: Acromegaly Consensus Group. Guidelines for acromegaly management: An update. J Clin Endocrinol Metab 94:1509-1517, 2009

22. Osamura RY, Kajiya $H$, Takei M, Egashira N, Tobita M, Takekoshi S, Teramoto A: Pathology of the human pituitary adenomas. Histochem Cell Biol 130:495-507, 2008

23. Pan LX, Chen ZP, Liuand YS, Zhao JH: Magnetic resonance imaging and biological markers in pituitary adenomas with invasion of the cavernous sinus space. J Neurooncol 74: 7176, 2005
24. Peak K, Kim SH, Song SH, Choi SW, Koh HS, Youm JY, Kim Y: Clinical significance of Ki 67 labeling index in pituitary macroadenoma. J Korean Med Sci 20:489-494, 2005

25. Plata AO, Suck MLT, Gomez ML, Heras A, Garcia AS: Study of the telomerase HTERT fraction, PCNA and CD34 expression on pituitary adenomas. Association with clinical and demographic characteristic. J Neurooncol 84:159-166, 2007

26. Rotondo F, Cusimano M, Scheithauer BW, Rotondo A, Syro LV, Kovacs K: Ghrelin immunoexpression in pituitary adenomas. Pituitary 14:318-322, 2011

27. Snow RB, Johnson CE, Morgello S, Lavyne MH, Patterson $\mathrm{RH}$ Jr: Is magnetic resonance imaging useful in guiding the operative approach to large pituitary tumors? Neurosurgery 26:801-803, 1990

28. Thapar K, Scheithauer BW, Kovacs K, Pernicone PJ, Laws ER Jr: p53 expression in pituitary adenomas and carcinomas: Correlation with invasiveness and tumor growth fractions. Neurosurgery 38:765-770, 1996

29. Wierinckx A, Auger C, Devauchelle P, Reynaud A, Chevallier P, Jan M, Perrin G, Fevre-Montage M, Rey C, Figarella-Branger D, Raverot G, Belin MF, Lachuer J, Trouillas J: A diagnostic marker set for invasion, proliferation, and aggressiveness of prolactin pituitary tumors endocrine-related. Cancer 14:887900, 2007 